Simpson GM, Lee JH, Zoubek B, Gardos G (1979): A rating scale for tandive dyskinesia. Psychopharmacology 64:171179.

Smeraldi E, Scorza-Smeraldi R (1976): Interference between anti-HLA antibodies and chlorpromazine. Nature 260:532-533.
Svejgaard A, Ryder LP (1976): Interaction of HLA molecules with non-immunological ligands as an explanation of HLA and disease. Lancet ii:547-549.

Teresaki PI (1970): In Brand DL, Rae JG (eds), Manual of Tissue Typing Techniques. Bethesda, MD: National Institutes of Health.

\section{Treatment of Catatonia with an Anticholinergic Agent}

To the Editor:

In their recent overview, "Benzodiazepines and Catatonia," Menza and Harris (1989) describe numerous theories regarding the response of catatonic symptoms to benzodiazepines. Among these, they state that, "[L]ow doses of GABA agonists reduce cholinergic activity in the basal ganglia," while, "[H]igher doses of GABA agonists reduce both dopaminergic and cholinergic activity." There is an analogy to the model of muscarinic-dopaminergic hyperactivity in schizophrenia proposed by Tandon and Greden (1989), where increased muscarinic activity has been implicated in the production of negative schizophrenic symptoms. Panzer et al (1990) have suggested that it may account for catatonia in some cases as well. Although cholinergic activity has previously beeii linked to catatonia (Van Andel 1958), Panzer et al reported the first case of a patient with schizoaffective disorder in whom catatonia was treated with an anticholinergic agent. We report the first case of catatonia in a patient with schizophrenia that was successfully treated with a parenteral anticholinergic agent.

Mr. A is a 24-year-old man whose psychiatric history began 9 months prior to his admission. His wife described him as becoming paranoid and increasingly hostile without provocation; he was afraid that his wife would take his two children away from him. The couple separated shortly thereafter, and the patient remained ambivalent about the marriage. Mr. A was al io using cocaine, marijuana, and alcohol at that time.

Two months prior to admission, the patient began experiencing auditory hallucinations, displayed loose associations and social withdrawal, and made a suicide attempt by pouring gasoline on his shirt and igniting it. Subsequent evaluation at a local hospital revealed normal laboratory values including electrolytes, thyroid function tests, VDRL, EEG, and computed tomography of the head without contrast. He was transferred to the University of Michigan Hospital, but left against medical advice the following day. According to his family, Mr. A's psychotic symptoms cleared somewhat over the next several days.

Shortly thereafter, however, his level of functioning again deteriorated. Mr. A quit his job of 2 years, and became more disorganized; he became withdrawn, his affect was flat, and his personal hygiene was poor. He had markedly decreased speech production with severe psychomotor retardation.

Upon readmission Mr. A was noted to have increased rigidity, heightened startle response, and an absence of spontaneous movements; he was essentiaily mute. Although muscle tone was increased, there was no "waxy flexibility." Laboratory values (including ceruloplasmin, VDRL, and thyroid function tests) again were normal except for ketones on urinalysis, and mildly elevated calcium, protein, albumin, and total bilirubin, all of which returned to normal within a few days without treatment. Urine toxicology screen was only posiitive tor dipienhydramine (which by our assay may persist in the urine for more than a week after the last dose.) Chest radiograph and magnetic resonance imaging of the head were both normal. Neurological consult concurred with our assessment of psychogenic catatonia (Fricchione 1985).

Intravenous hydration was begun as the patient was unable to take anything by muvih. After $24 \mathrm{hr}$ of hydration without improvement, the patient was given $2 \mathrm{mg}$ of benztropine intravenously in an attempt to treat his catatonia. There was minimal response to the initial dose, and another $2 \mathrm{mg}$ was given $1 \mathrm{hr}$ later. Within $60 \mathrm{~min}$, the patient was able to answer 
questions readily. He would initially only speak in one or two word sentences, but progressively became more verbal. He was gradually able to attend to his persor:al hygiene, feed hirnself, and walk around the unit with assistance; these changes occurred over the course of $\mathbf{2}$ days. Mr. A still complained of auditory hallucinations at this time. He was subsequently begun on thiothixene and benztropine and continued to improve. Within several days he denied any hallucinations or delusions, but remained somewhat guarded. There was no recurrence of catatonic symptoms during the :emainder of his hospitalization, and the patient was discharged after 2 weeks in good condition with the diagnoses of schizophrenia and polysubstance abuse.

The rapid response of catatonic symptoms in this first-break, medication-naive patient to a parenteral anticholinergic agent lends support to the theory that a disproportionately high degree of cholinergic tone may be responsible for the observed catatonic symptoms. As this is only the second case reported by our institution (Panzer et al 1990), a cautious interpretation of these data is required. Clearly, future controlled studies are in order. Nonetheless, our findings support the conclusions of Menza and Harris regarding a possible anticholinergic mechanism of benzodiazepine treatment of catatonia. An increase in cnolinergic activity may be one mechanism underlying disorders such as negative schizophrenia symptoms and catatonia.

\section{Ronald C. Albucher John DeQuardo Rajiv Tandon}

Department of Psychiatry

University of Michigan Medical Center

Ann Arbor, MI 48109-0120

\section{References}

Fricchione GL (1985): Neuroleptic catatonia and its relationship to $\mu$ sychogenic catatonia. Biol Psychiatry 20:304 313.

Menza MA. Harris D (1989): Benzodiazepines and catatonia: An overview. Biol Psychiatry 26:842-846.

Panzer M, Tandon R, Greden JF (1990): Benzodiazepines and catatonia. Biol Psychiatry 28:178-179. (Correspondence.)

Tandon R, Greden JF (1989): Cholinergic hyperactivity and negative schizophrenic symptoms: A model of dopaminergic/cholinergic interactions in schizophrenia. Arch Gen Psychiatry 46:745-753.

Van Andel H (1958): Neuropharmacological studies on catatonic phenomena: In: Bradley PB, Deniker P, Padouco-Thomas C (eds), Neuropsychopharmacology. Amsterdam: Elsevier, pp 701-703.

\section{Response}

To the Editor:

This report by Drs. Albucher, DeQuardo, and Tandon is now the second report in which a patient with a "psychogenic" catatonia appears to have responded to an anticholinergic agent. As they point out, and as we have speculated in the past, this response may be relevant to the widely reported usefulness of the benzodiazepines in catatonia (Menza and Harris 1989).

Assuming that this response is reproducible under more controlled conditions, there are a number of possible implications. The model of catatonia as a dopaminergic/cholinergic imbalance fits nicely with these case reports but one nust be careful in making too much of very little sata. The data are also consistent with our sfeculations concerning crmplex partial seizures activity, because limbic electrical discharges are suppressed by anticholinergic agents (Meldrum 1984). Whatever the mechanism, we look forward to further corifuilicu diati on anticholinergic ageh.s and catatonia.

\section{Matthew A. Menza}

Department of Psychiatry

UMDNJ

Robert Wood Johnson Medical School

New Brunswick, NJ 08903

\section{References}

Meldrum B (1984): Anticonvulsants and the limbic system. In Trumble MR, Zarifian E (eds), Psychopharmacology of the Limbic System. Oxford: Oxford University Press, p 96.

Menza MA, Harris D (1989): Benzodiazepines and Catatonia: An Overview. Biol Psychiatry 26:842-846. 